\title{
Model Driven Design of Web Service Operations using Web Engineering Practices*
}

\author{
Marta Ruiz, Vicente Pelechano \\ Universidad Politécnica de Valencia \\ Camí de Vera s/n, Valencia-46022, España \\ \{mruiz, pele\}@dsic.upv.es
}

\begin{abstract}
The design of Web Services is nowadays emerging as one of the most important tasks in the development of a Service Oriented Application. Web service designers need some guidelines to achieve a design of quality. In this paper we provide a methodological guide in the context of a Web engineering method called OOWS. Our approach allows identifying the operations of Web services following a model driven approach, taking the OOMethod / OOWS conceptual models as the source. To document our approach, we apply our ideas to a real case study of a Web application to manage University Research Groups.
\end{abstract}

Keywords: Web services design, Service Oriented Architecture, Model Driven, Web engineering, Software Process Engineering.

\section{Introduction}

Web services have emerged as important components of modern Web applications. There are some approaches working on designing services interfaces. Ambler [1] presents several steps for deriving a set of Web services from an object-oriented application, identifying domain packages and the services that each package provides. Papazoglou [8] describes a design methodology for Web services based on business processes. On the other hand, Web engineering methods are extending their proposals to introduce Web services into Web conceptual models. In this context, we can distinguish approaches that introduce some kind of syntactic mechanism to include Web services calls into navigational models like OOHDM [13] and UMLGuide [3]. However, these approaches do not give support to the design and development of Web services. Other methods like OO-H [4] allow Web developers to generate Web services interfaces but they neither give support to their design. Finally WebML [5] captures Web services invocations by means of visual representations. They describe the Web interactions using hypertext models but do not follow any strategy or guide to design Web services.

\footnotetext{
* This work has been developed with the support of MEC under the project DESTINO TIN2004-03534, cofinanced by FEDER and the PAID-04-06 project by UPV.
} 


\section{Marta Ruiz, Vicente Pelechano}

In this work, we present a Web service design guide that extends the OOWS method [10]. OOWS is a Web engineering method that is based on the principles defined by de Model-Driven Development (MDD) [6]. It allows us to automatically obtain fully operative web applications from conceptual models. In order to design the operations of Web services, we consider that the OO-Method [9] / OOWS models are a key point. OO-Method / OOWS models allow us to automatically obtain Web services operations.

The main contributions of this work are:

- Determining which models are useful to obtain Web services operations.

- Proposing a methodological guide to design the operations of Web services.

- Identifying operations that give support to the functional requirements of an application, user identification and management, information retrieval, navigation and presentation. A first approach of this work can be found in [12].

- Providing a Web services design method with tool support to automatically generate the WSDL of the Web services.

The structure of the paper is the following: section 2 introduces a Web service design approach based on the OO-Method / OOWS method. Section 3 presents a methodological guide to obtain well designed Web services in a SOA. In our approach, a set of operations are identified taking the OO-Method / OOWS conceptual models. These operations define the public operations that a Web service can offer to web applications (major web clients). Finally, we present some conclusions and further work in section 4.

\section{An overview of the Web service design process}

In this section, we introduce a Web service design process that takes into account the OO-Method / OOWS models. To present this process we use the notation and terms defined in the Software Process Engineering Metamodel (SPEM) proposed by the OMG [14]. SPEM is a meta-model for defining software engineering process models and their components. First, we present the Disciplines that define our process as well as the Activities, WorkProducts and ProcessRoles that are included in each step. Next, we present the Sequencing of Activities that defines the design process.

\subsection{Web Service Design Process}

According to SPEM, an Activity is a piece of work performed by one ProcessRole in order to obtain a WorkProduct. A Discipline partitions the Activities within a process according to a common "theme".

Our Web service design process is defined from the following disciplines: Requirements Elicitation (see figure 1-A), OO-Method (see figure 1-B) and OOWS (see figure 1-C) conceptual modeling and code generation. 


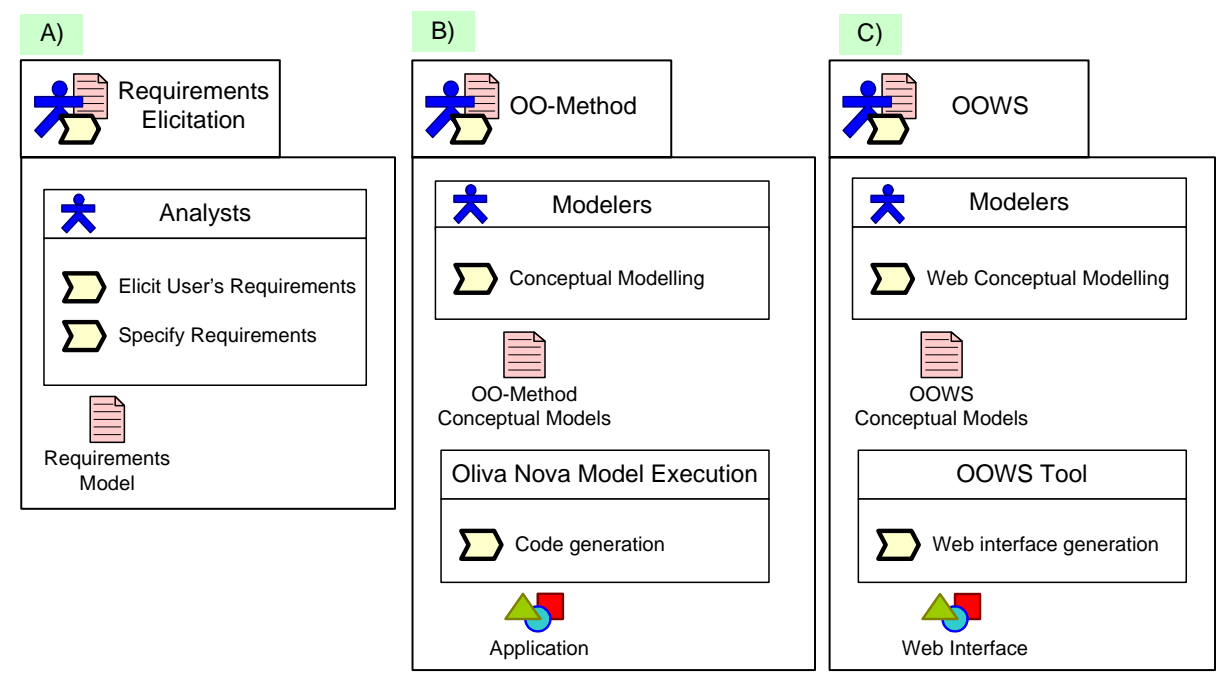

Fig. 1. Requirements Elicitation, OO-Method and OOWS Disciplines

\subsubsection{Requirements Elicitation.}

This discipline includes those activities that are related to the elicitation of the user's requirements. These activities are two (see figure 1-A): (1) elicit the user's requirements and (2) specify requirements. The activities must be performed by Analysts.

The WorkProduct that the analysts must obtain after performing the activities is a requirements model: one task diagram for each kind of user, and a textual and a graphical description for each leaf task. Figure 1-A shows the definition of this discipline by means of the notation proposed in the SPEM.

\subsubsection{The OO-Method.}

This discipline includes those activities that are related to the model and generation of applications. These activities are based on the development process of the OOMethod [9] and its strategy of automatic code generation. Nowadays, the OO-Method approach has an industry-oriented implementation called OlivaNova Model Execution (ONME) [7] that has been developed by CARE Technologies S.A.

Thus, the activities of this discipline are two (see figure 1-B): (1) define a conceptual schema to represent the application requirements (from the requirements model defined by analysts); and (2) generate code from the conceptual models that implements an application. The first activity must be performed by OO-Method modelers, and the WorkProduct that must obtain is a set of conceptual models: class, dynamic (state transition and sequence diagrams) and functional models. The second 
activity must be performed by ONME and the WorkProduct that must obtain is the final application.

\subsubsection{OOWS.}

This discipline includes those activities that are related to the generation of a Web application. These activities are based on the development process proposed by the OOWS method [10] and its strategy of automatic code generation. Thus, the activities of this discipline are two (see figure 1-C): (1) defines the conceptual models of the Web application (from the requirements and the class model defined previously); and (2) generates the Web interface from the conceptual models that implements the Web functionality of the application generated by the OO-Method discipline.

The first activity must be performed by OOWS modelers, and the WorkProduct that must obtain is a set of conceptual models (user, navigation and presentation models). The second activity must be performed by the OOWS tool and the WorkProduct that must obtain is a Web interface.

In order to clearly show how these activities should be performed and which the relationships among them are, the next subsection introduces the activity sequencing. This sequencing describes both the order in which the different activities within our process must be performed and the input and output WorkProducts of each activity.

\subsection{Sequencing of Activities}

Figure 2 shows the activity sequencing of our process (following the activity diagram notation proposed by SPEM). According to this figure, our Web service design process is defined as follows: first, analysts create a requirements model after analyzing the user's needs. Then, on the one hand the OO-Method modelers define the OO-Method conceptual models of the application from the requirements, and the ONME tool automatically generates a software application. On the other hand, OOWS modelers model the Web application and then the OOWS tool generates the Web interface of this application. Finally, the operations of the Web service are designed.

As we can see, there are dependencies between activities: the OO-Method modelers need the requirements models to model the application; the OOWS modelers need the requirements and the OO-Method models to model the Web interface; and the Web service designer needs the requirements models, the class models of the OO-Method discipline, and the user, navigation and presentation models of the OOWS discipline to identify the operations of the Web service to be published.

In the next section, we introduce a guide to support Web service designers in the achievement of their activity. 


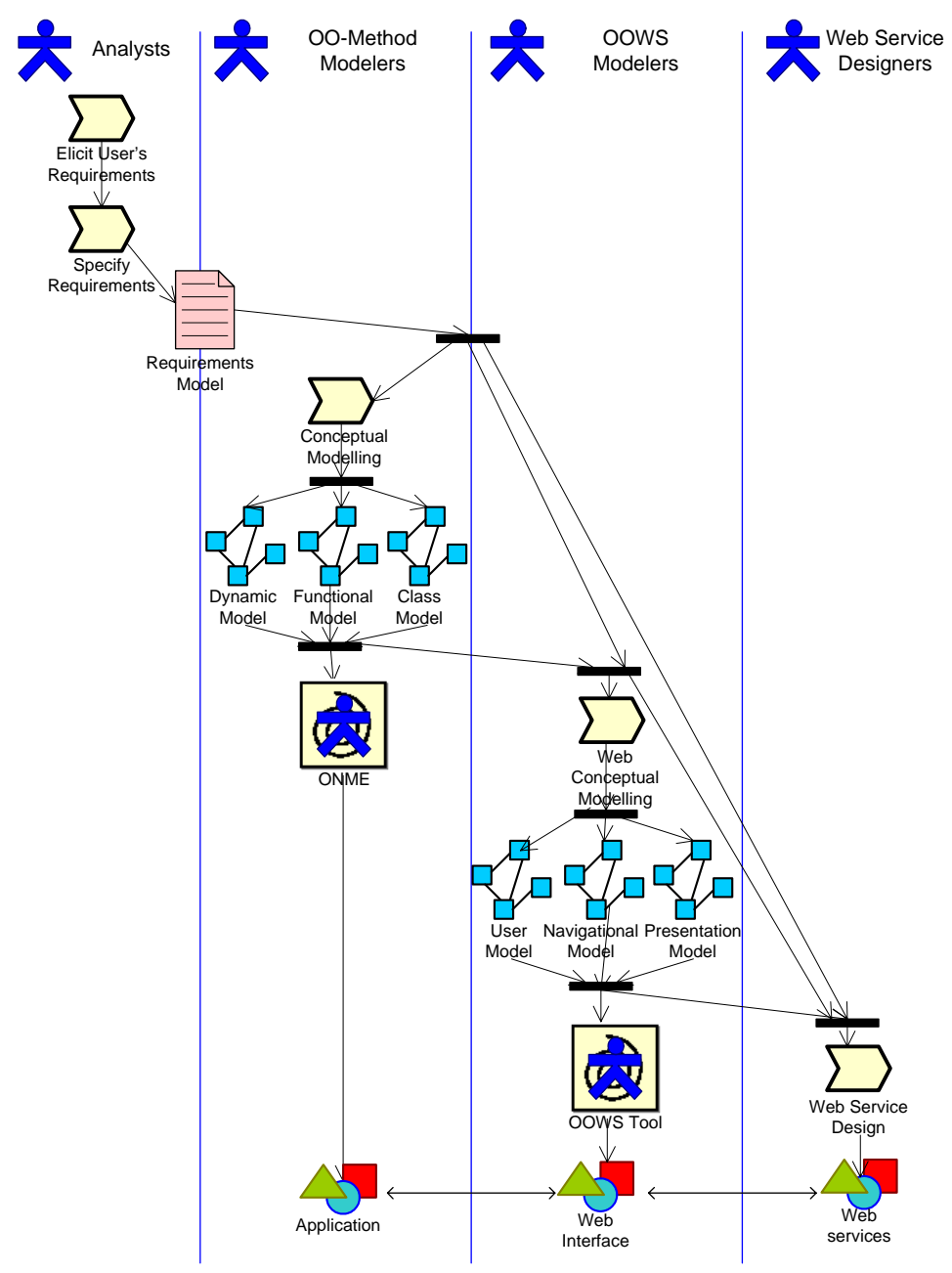

Fig. 2. Activity sequencing

\section{The Web service design activity}

In this section we present a methodological guide to obtain, in a systematic way, the operations that implement the requirements of a Web application in a SOA. The operations that are related to the integration with third party systems are out of the scope of this work. Information about this can be found in [15]. 


\subsection{Identifying operations from Analysts' WorkProduct}

The WorkProduct that analysts must obtain after performing the activities is a requirements model. The requirements model is based on the concept of task [16]. This model is built in two main steps: (1) first, analysts must define a task taxonomy for each kind of user that can interact with the system, where tasks are decomposed into subtasks by following structural or temporal refinements; (2) next, each task is described by analyzing the interaction that users require from the system to achieve each tasks.

The operations detected from the task diagram provide operations to implement the functional requirements of a system. A task diagram identifies user goals and the activity that a user performs to achieve these goals. Therefore, we think that this diagram can help us to identify the public operations that must offer our service in order to build Web applications that completely support user tasks. The potential "users" can be persons, Web pages or other external applications.

We present a set of steps that can be followed to automatically identify the operations. In the first place, we should traverse the task diagram paying attention to two kind of tasks that are going to be selected as candidate operations to be published: (1) those leaf tasks that not participate in a structural relationship (represented by solid arrows between a task and their subtasks); and (2) those tasks that are parents of a structural relationship.

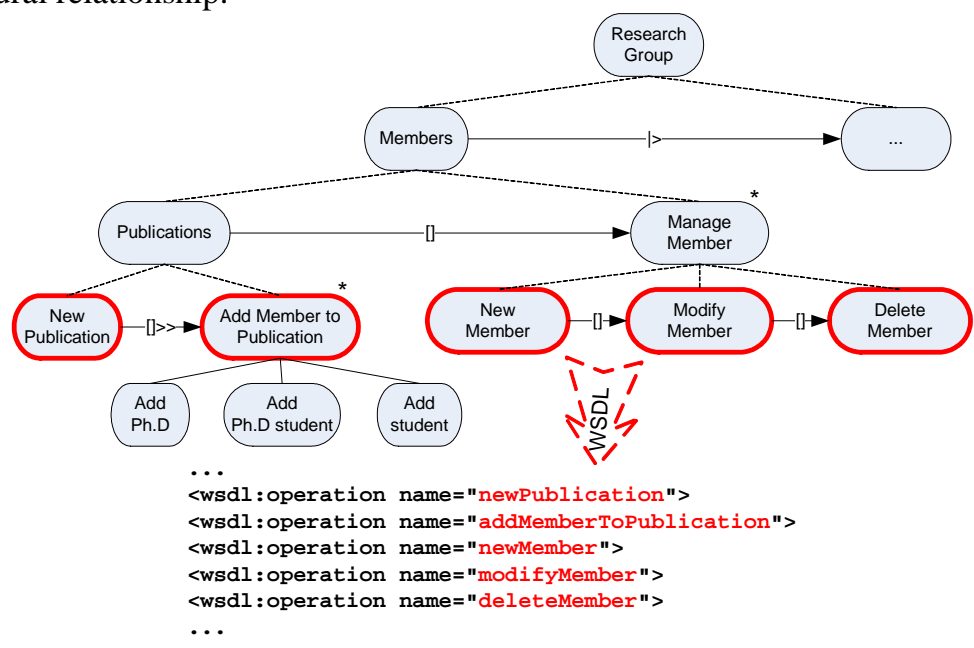

Fig. 3. Operations detected in the task diagram

In figure 3 we have a partial view of the description of how a researcher can manage a research group. Following the steps previously presented, we define the next operations: newPublication, addMemberToPublication, newMember, modifyMember and deleteMember.

Each task has associated: (1) a textual description that define the goals and the users that can achieve this task; and (2) a graphical description by means of activity diagrams. 
Operations arguments should be detected from the graphical descriptions associated to tasks. In the graphical description, each node of the activity diagram defines: (1) a system action (stereotyped with $<<$ function $>>$ or $<<$ search $>>$ keyword); or (2) an interaction point (IP) (nodes stereotyped with $<<$ input $>>$ or $<<$ output $>>$ keyword). Each IP define an instant in the achievement of a task where the user interacts with the system. In this context, the system actions performed after a $<<$ output $>>$ IP, allow Web services designers to identify the entity involved in the task. Thus, using this $<<$ output $>>$ IP and the $<<$ input $>>$ IP from the graphical description together with the class diagram (a WorkProduct of the OO-Method modelers), we can obtain the arguments of each operation. We detect each participant class in an operation by matching the entities that are specified in the node with the class diagram. For each identified class we add its type to the arguments of the operation detected from this branch of the task tree.

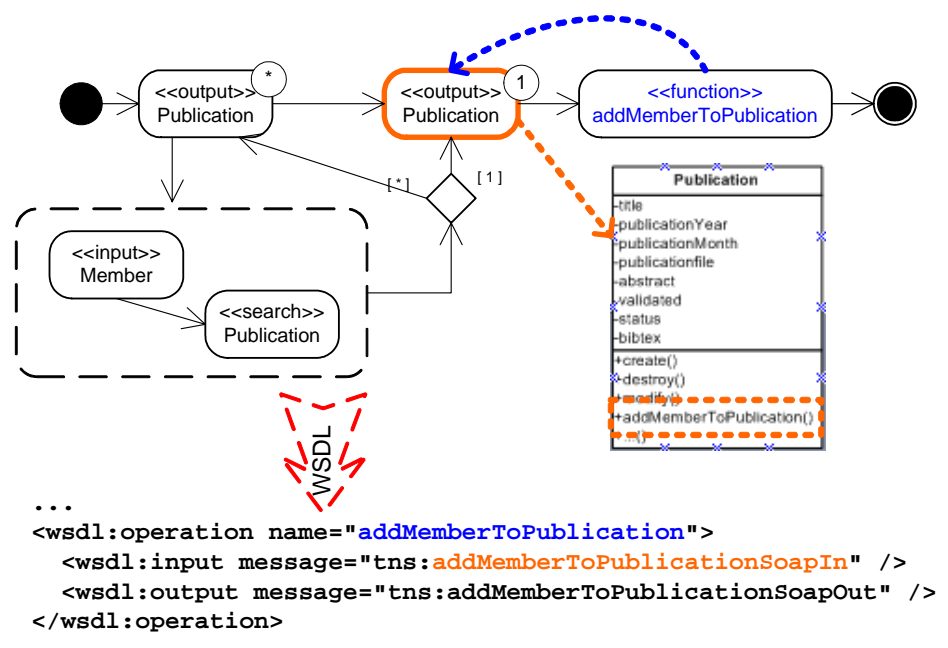

Fig. 4. Arguments of addMemberToPublication

Figure 4 shows the arguments for the operation addMemberToPublication that has been previously defined. This operation is detected from the task Add Member to Publication, so the arguments of this operation are taken from the graphical description of this task. This graphical description has the <<output $>>$ IP Publication which maps with the Publication class in the class diagram. Matching the addMemberToPublication task with the function of the Publication class that is associated, we obtain the arguments for our Web service operation. So we add the argument of Member type to the arguments of the operation addMemberToPublication.

Figure 5 shows the Web page that gives support the execution of the addMemberToPublication operation. 


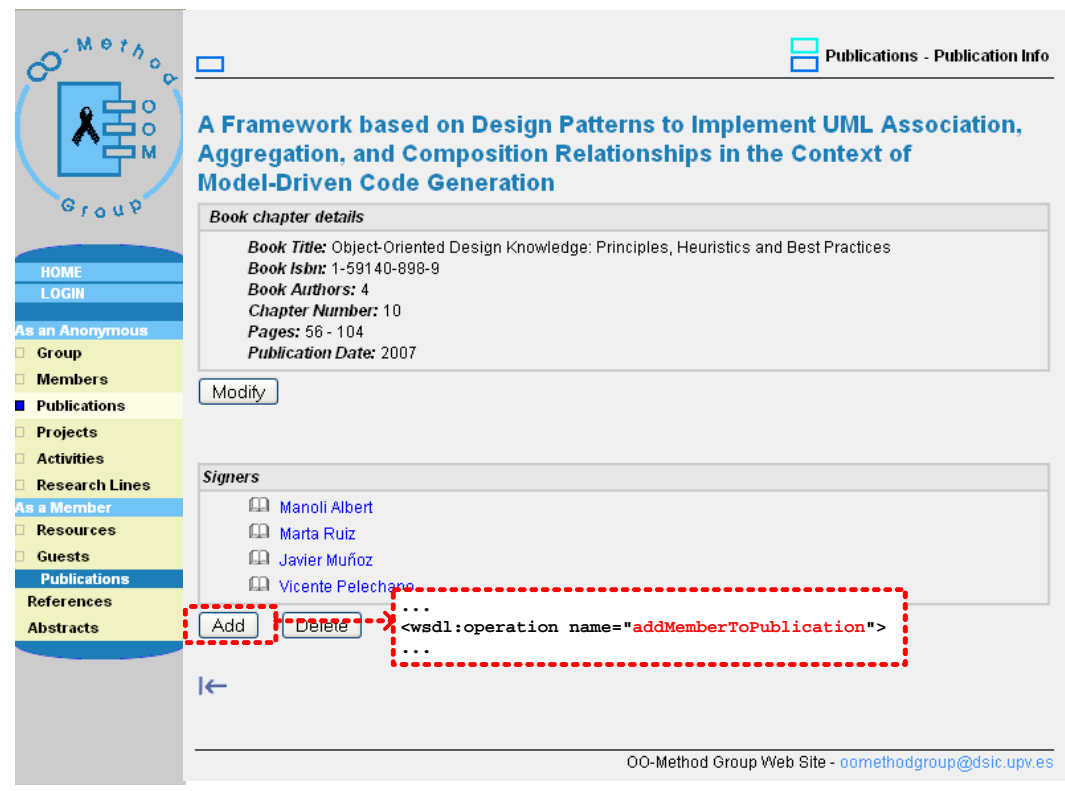

Fig. 5. The implementation of addMemberToPublication

\subsection{Identifying operations from OOWS modelers' WorkProduct}

The WorkProduct that OOWS modelers must obtain is a set of conceptual models (users, navigation and presentation models), that give support to the tasks identify by analysts. These models allow (1) expressing what kind of users can interact with the system and what system visibility they can have; (2) defining the navigational semantics of the system; and (3) specifying its presentational requirements.

\subsubsection{User Model.}

The OOWS user diagram is used to detect kinds of users (roles) and assign to them permissions to interact with the system, providing a role-based access control (RBAC) [2]. The RBAC model gives us a guideline to perform the access control in an application. This model requires a minimum of five elements: users, roles, objects, operations and permissions. This model also provides functions to define the functional specifications.

Afterwards, the operations of this group are detected from both the user diagram and the RBAC model and are classified into three types:

- Those that provide support for the user identification: loginUser, logoutUser, obtainRol, changeRol and remindPassword.

- Those that give support for the generic user administration: newUser, modifyuser, deleteuser. 
- Those that give support to the management of user's permissions and roles: newRol, deleteRol, addUserToRol, removeUserToRol, addPermission and removePermission inherited from the RBAC model

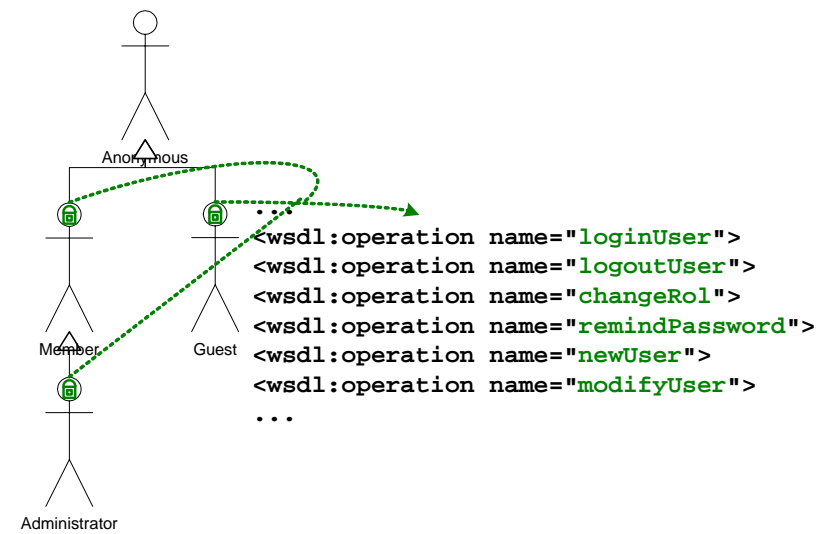

Fig. 6. WSDL based on the User Diagram

It is not necessary to publish all this functionality for every implementation. So the Web service designer must decide which operations should be published. We implement the following six operations in our running example (see figure 6): loginUser, logoutUser, changeRol, remindPassword, newUser and modifyuser.

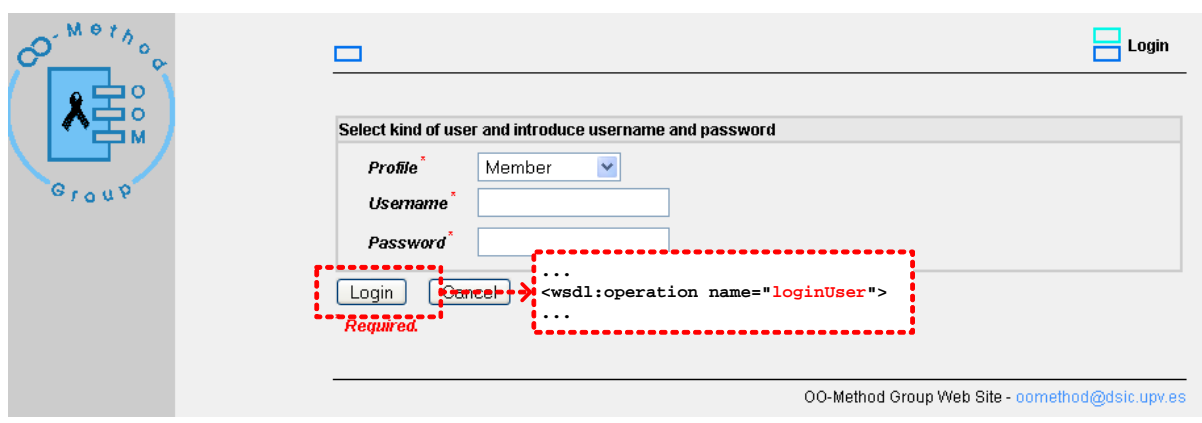

Fig. 7. The implementation of loginUser

Figure 7 shows the Web page that gives support the execution of the loginUser operation.

\subsubsection{Navigational Model.}

Once users have been identified, a structured and organized system view for each user type must be specified. These views are defined over the Class Diagram, in terms of the visibility of class attributes, operations and relationships. This model is built in 
two steps: (1) first, a global view over the navigation is defined (Navigational maps); and (2) a detailed description of the elements defined in the previous step is performed (Navigational Contexts).

The navigational map is depicted by means of a directed graph whose nodes represent navigational contexts and its arcs represent navigational links that define the valid navigational paths over the system. The operations that define this primitive allow the reuse of navigation facilitating the implementation of adaptation and personalization mechanisms. In this context, a proposal for describing Adaptive LinkHiding techniques in base of the description of Navigational Links has been introduced in [11]. This group has two operations (see figure 8-A), one for each kind of navigational link:

1. The explorationLink operation gives support for the implementation of the exploration links (represented by dashed arrows). They can be activated from any context of the navigational map providing access to the context where the link ends. It returns the page links to those reachable navigational contexts through an exploration link.

2. The sequenceLink (context) gives support for the implementation of the sequence links (represented by solid arrows). They represent a reachability relationship between two contexts. This kind of link can be activated from the context that defines the link source and provide access to the context where the link ends. It returns the set of page links of the navigational contexts accessible by the user from a specific context.

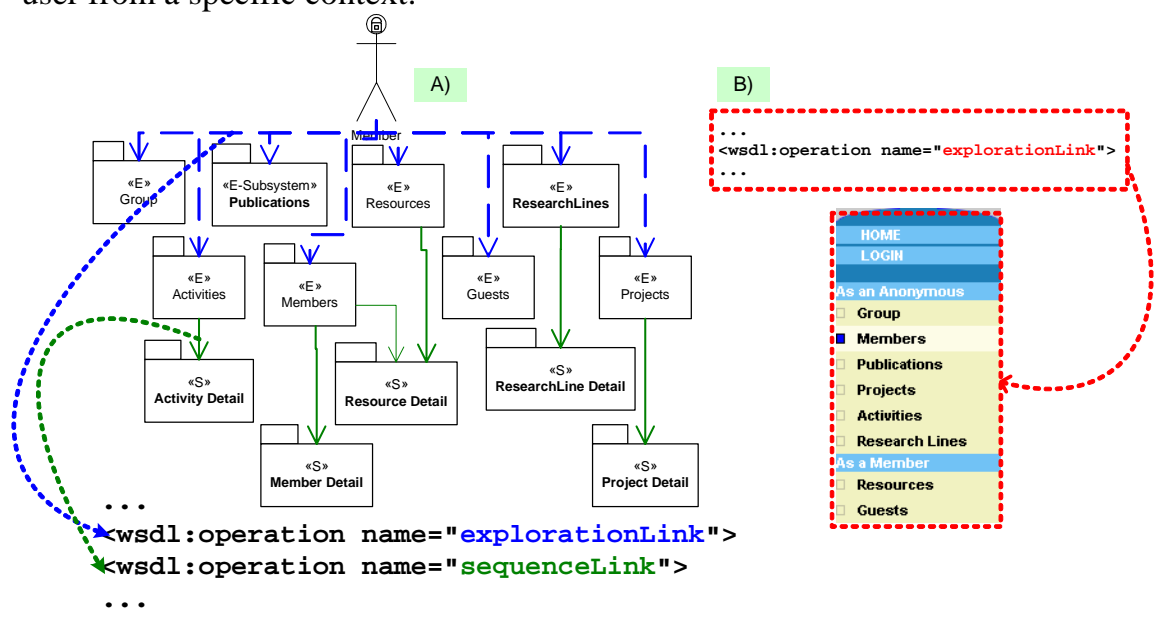

Fig. 8. Operations detected from the Client navigational map and the implementation of the explorationLink operation

Figure 8-B shows the Web page where the result of the call of the explorationLink operation is shown. This operation returns the page links (contexts) that are reachable from somewhere of the Web application.

The navigational context defines operations to retrieve the information that must be shown in each navigational context (a web page in the running example). For each navigational context, we can define (see figure 9): 
1. The retrieveViewName ([attributeID]) operation allows us to obtain the information specified in the navigational context views. This operation returns (1) a set of instances or (2) a specific instance of the classes defined in the context view. We define an operation of this kind for each view defined in a navigational context. Figure 9 shows the operation detected from the navigational context Members: retrieveMembers.

2. The getIndexedIndexName (attributes) operation gives support for the index mechanisms. They provide an indexed access to the population of objects. This operation returns the list of the resumed information defined in a given index allowing the user to choose one item (instance) from the list. It is defined for every index in a navigational context. An example of this operation is shown in figure 9, where the operation getIndexedMember is identified from the index of the context Members.

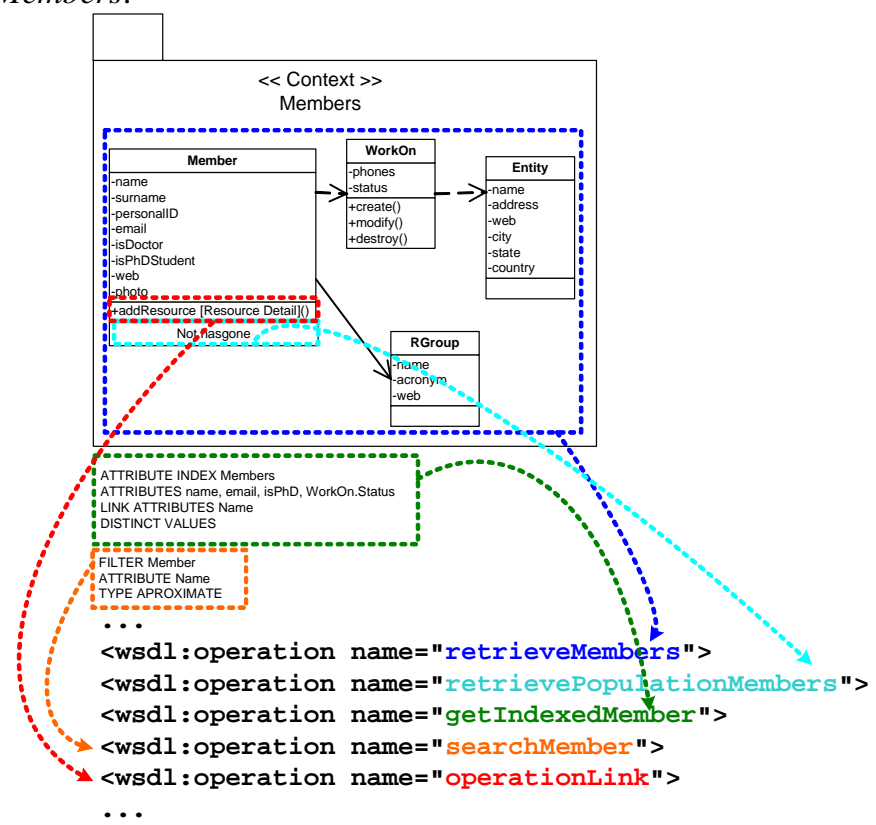

Fig. 9. Operations detected from the navigational context Members

3. The retrievePopulationViewName (attribute*) operation gives support to population condition mechanisms. They define an object retrieval condition that must be satisfied. This condition can be specified to any navigational class. The operation can need one or more attributes, depending on the definition of the population filter. This operation returns a set of instances of the class where the population filter is defined that fulfil the condition specified by the user. We define an operation of this kind for each view defined in a navigational context that has defined a population filter. Figure 9 shows the operation detected from the navigational context Members: retrievePopulationMembers.

4. The searchFilterName (value) operation gives support to search filters mechanisms. They allow filtering the space of objects that retrieve the navigational 
context. This operation returns the set of instances of the manager class (and its complementary classes) that fulfil the search conditions specified by the user. An operation is obtained for each filter of a navigational context. Figure 9 shows the detection of the operation searchMember from the filter defined in the context Member.

Moreover, the navigational map can also provide operations that support part of the implementation of the navigation defined in the navigational model.

5. The operationLink (service) operation gives support to the implementation of the operation links. Operation links represent the target navigational context that the user will reach after an operation execution. This operation returns the context (Web page) that the user accesses before one specific operation is activated. Figure 9 shows operationLink detected from the operation AddResource defined in the class Product.

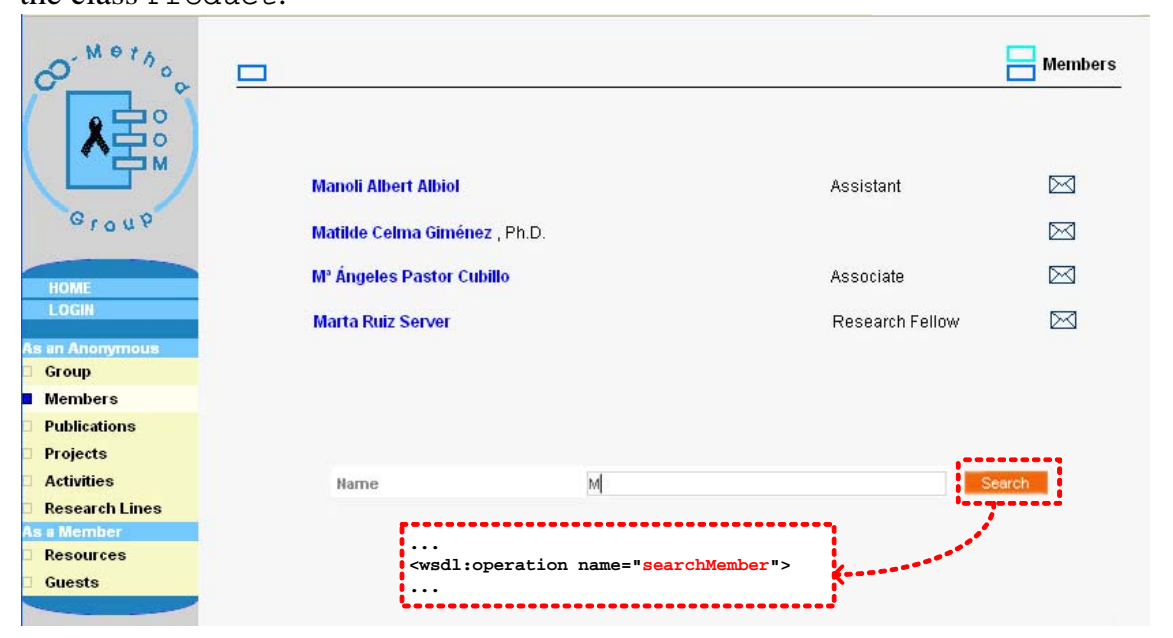

Fig. 10. The implementation of searchMember

Figure 10 shows a Web page in which the result of the operation searchMember can be seen (defined from the filter of the Member Context in figure 9). In this case, the filter condition is that the name of the member has the letter " $M$ ". Then, the web page of this figure shows all members related with "M".

\subsubsection{Presentation Model.}

Once the navigational model is built, modelers must specify presentational requirements of web applications using a presentation model. It is strongly based on the navigational model and it uses its navigational contexts to define the presentation properties. Presentation requirements are specified by means of patterns that are associated to the primitives of the navigational context. The basic presentation patterns are:

- Information Paging. This pattern allows defining information "scrolling". All the instances are "broken" into "logical blocks", so that only one block is visible at a 
time. Mechanisms to move forward or backward are provided. The required information is: (1) cardinality represents the number of instances that make a block; and (2) access mode (sequential or random).

- Ordering Criteria. This pattern defines a class population ordering (ASCendant or DESCen-dant).

- Information Layout. OOWS provides 4 basic layout patterns: register, tabular, master-detail and tree.

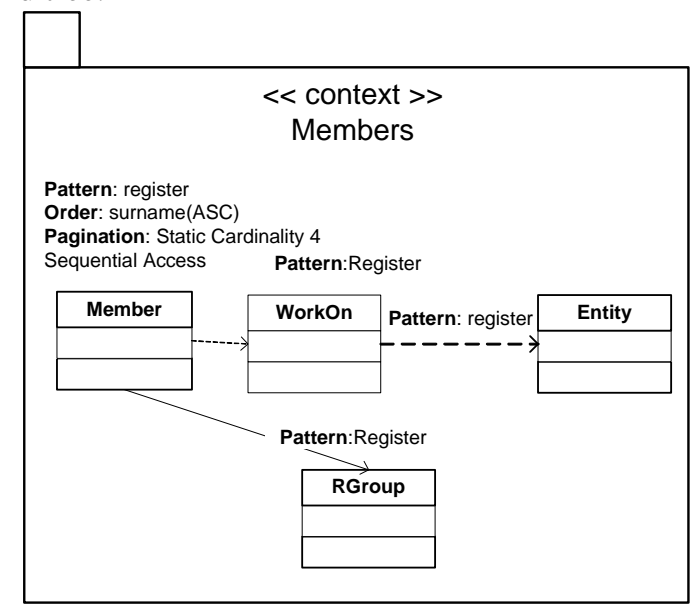

Fig. 11. Members Context Presentation Model

Figure 11 shows the presentation requirements specified for the Members Navigational Context. According to these requirements the list of members provided by the context is shown in a register format. This list is grouped in blocks of four members. The surname of each member is used to order them in an increase way.

These presentation patterns, together with the specified navigation features, capture the essential requirements for the construction of web interfaces. Thus, Web service designers can identify two operations from this model:

1. The presentationInfo (operation, operationAttributes, pagination, order, patter) operation, where operation is an operation published by the Web service, operationAttributes is the set of attributes of that operation, and pagination, order and patter are the values for the presentation patterns. This operation returns a piece of Web page with the information retrieved presented with the values defined by the user at the call.

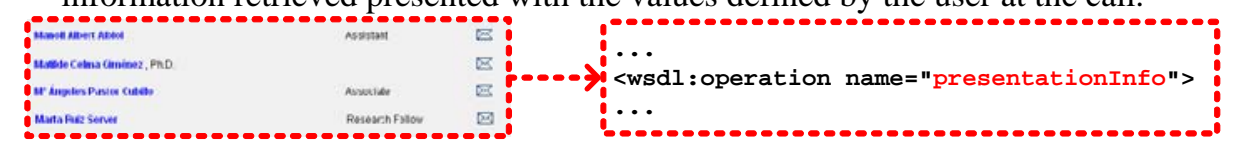

Fig. 12. The result of presentationInfo (searchMember)

Figure 12 shows the result of the operation searchMember. In this case, the result must be presented as a piece of a Web page where the products are showed in register, in groups of three elements and ordered by price increasing. 
2. The operation presentationContext (context) returns a Web page created for the context attribute. This Web page follows the presentation patterns indicated in the presentation model. To create the Web page, this operation uses some the operations presented in this article: retrieveViewName for each view defined in the context and explorationLink and sequenceLink for the link pages. Moreover, if the context has some filter or index, then the Web page will show these operations.

Figure 13 shows the Web page created as a result of the presentationcontext(Members) operation. The information retrieved from the Members view is showed in register, in groups of four and ordered by surname increasing. In addition, it shows links to those pages reachable since this context.

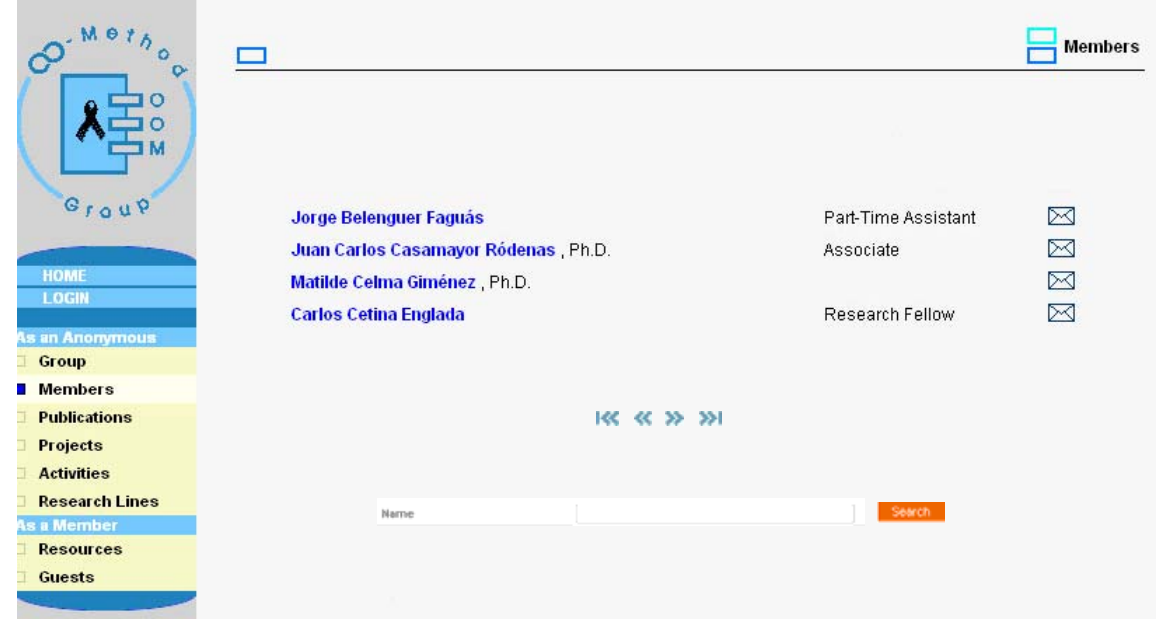

Fig. 13. presentationContext (Members)

\subsection{Tool support}

In this section, we present a tool that helps Web service designers in the identification and design of the Web services operations. This tool also allows Web service designers to associate a WSDL specification to a specific application. This tool, which is called ITOW, automatically generates the WSDL specification of the application modeled by the ONME and OOWS CASE tools (see figure 14).

ITOW provides an easy-to-use graphical interface. Before ITOW generates the WSDL specification, it is necessary to configure the following properties:

- Load Project: Defines the XML file where the OO-Method and the OOWS models are stored.

- Save Project: Defines the target location where the generated WSDL document will be stored.

- URL: Defines the namespace of the Web application to be included in the WSDL file. 
- Kind of WSDL: Specifies if the kind of WSDL file (document or RPC) that is going to be generated.

- Generate: Indicates if the WSDL document has already been generated or not.

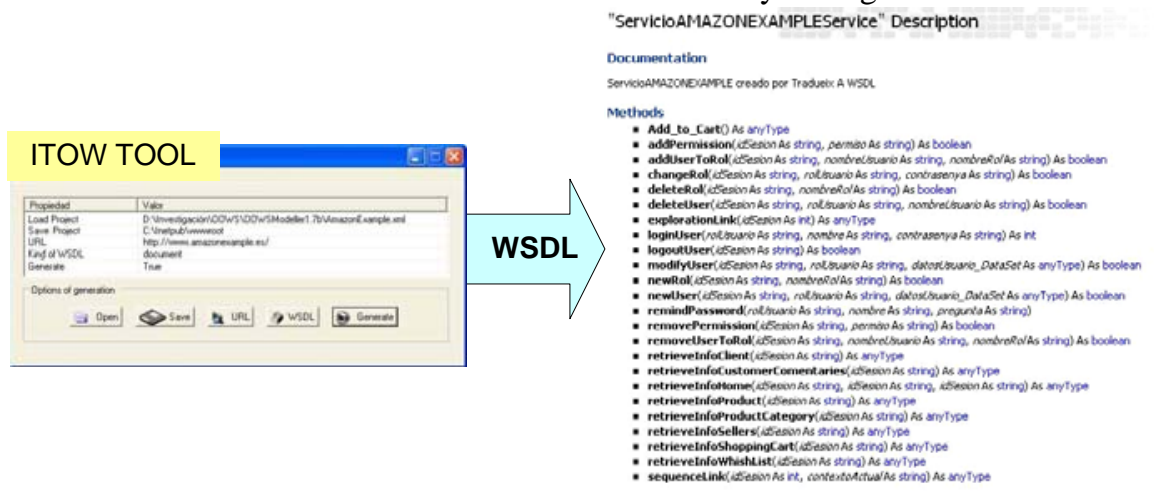

Fig. 14. Steps in the automatic generation of WSDL

Once we have configured these properties, the ITOW tool starts the transformation process to obtain the WSDL document that defines Web services. This transformation process follows the approach presented in this work.

\section{Conclusions and Further Work}

This article has presented an approach to introduce SOA and the Web services technology as an extension of the OO-Method / OOWS method. We have presented a methodological guide to help Web service designers to obtain the operations that define the Web services from models. This guide can be generalized to other Web Engineering Methods, because the OO-Method / OOWS method shares with them the most common models and primitives taken as source to obtain the Web services.

The ideas presented in this work have been already applied to the development of several applications like the Web site http://oomethod.dsic.upv.es, which supports the management of our research group and in the intranet management system for the General Library of the Technical University of Valencia. Nowadays, we are developing an e-commerce system and a travel agency. These two systems, in addition to provide Web services, integrate functionality of third parties applications.

Several extensions to this work are under development. We are working on integrating this approach on the ONME tool [7]. For this purpose, we are analyzing the models provided by OO-Method / OOWS methods. Our intention is determine how operations presented in this work can be implemented from the functionality generated by the ONME tool. This allows us to provide a Web service with fully operative operations.

We are also working on providing mechanisms that facilitate the integration of Web applications with Third parties systems at the conceptual level [15]. When third parties provide us their functionality as Web services, we proceed to the Web service composition to achieve integration. 
We have used the presentation model defined within the OOWS approach to enrich the code generation process providing Web services that include presentational aspects. Our final objective is to generate reusable Web functionality that provides relevant information (content and presentation) to potential consumers [11].

\section{References}

[1] Ambler, S.W., Deriving Web services from UML models, Part 1: Establishing the process. http://www-106.ibm.com/developerworks/webservices/library/ws-uml1/, March 1, 2002.

[2] ANSI. Incits 359 (2004). American National Standard for Information technology. RoleBased Access Control.

[3] Dolog, P., Model-driven navigation design for semantic web applications with the UMLguide. In Maristella Matera and Sara Comai (eds.), Engineering Advanced Web Applications. (2004)

[4] Gómez, J., \& Cachero, C. , OO-H Method: extending UML to model web interfaces. In information Modeling For internet Applications. P. van Bommel, Ed. Idea Group Publishing, Hershey, PA, (2003). pp. 144-173.

[5] Manolescu, I., Brambilla, M., Ceri, S., Comai, S., \& Fraternali, P., Model-driven design and deployment of service-enabled web applications. ACM Trans. Inter. Tech. 5, 3, (2005) pp. 439-479.

[6] Mellor, S.J., Clark, A.N., Futagami, T.: Model-driven development - Guest editor's introduction. IEEE Software, 20 (5):14- 18, Sept.-Oct. 2003.

[7] OlivaNova Model Execution System. CARE Technologies. Retrieve November 3, 2005, from: http://www.care-t.com.

[8] Papazoglou, M.P. and Yang, J. Design methodology for Web services and business processes. In Proceedings of the 3rd VLDB-TES workshop (Hong Kong, August, 2002).

Springer, 2002, 54-64.

[9] Pastor, O., Gomez, J., Insfran, E., \& Pelechano, V. : The OO-Method approach for information systems modelling: from Object-Oriented conceptual modeling to automated programming. Information Systems 26, (2001).pp 507-534.

[10] Pastor, O., Fons, J., Pelechano, V., \& Abrahão, S. Conceptual modelling of Web applications: the OOWS approach, book chapter in Web Engineering - Theory and Practice of Metrics and Measurement for Web Development, Mendes E. (Eds.), Springer 2005, (2005). pp. 277-302.

[11] Rojas, G., Pelechano, V., \& Fons, J.: A Model-Driven Approach to include Adaptive Navigational Techniques in Web Applications. V International Workshop on Web Oriented Software Technologies - IWWOST, Porto, Portugal. (2005).

[12] Ruiz, M., Valderas, P. \& Pelechano, V.: “Applying a Web Engineering Method to Design Web Services”. 6th International Conference on Service Oriented Computing (ICSOC). Lecture Notes in Computer Science Vol. 3826/2005. (2005).

[13] Schwabe, D., Rossi, G. \& Barbosa, D.J.: Systematic hypermedia application design with OOHDM. Proc. ACM Conference on Hypertext. (1996). pp.166.

[14] Software Process Engineering Metamodel, version 1.1. Object Management Group. http://www.omg.org/technology/ documents/formal/spem.htm

[15] Torres, V., Pelechano, V., Ruiz, M., \& Valderas, P.: A model driven approach for the integration of external functionality in Web applications. The Travel Agency System. Workshop on Model-driven Web Engineering (MDWE). (2005).

[16] Valderas, P., Fons, J., Pelechano, V.: Developing E-Commerce Application from TaskBased Descriptions. 6th International Conference on Electronic Commerce and Web Technologies (EC-WEB). (2005). 